\title{
* Aspectos clínicos de la nueva píldora con gestodeno
}

\author{
Jaime Urdinola*; Rolf Alwers**
}

RESUMEN: Se realizó un estudio abierto, prospectivo y multicéntrico en Colombia con el objeto de evaluar la eficacia, la seguridad anticonceptiva, el control del ciclo y los efectos secundarios de la nueva píldora anticonceptiva con $75 \mu \mathrm{g}$ de gestodeno y $30 \mu \mathrm{g}$ de etinilestradiol durante 12 ciclos consecutivos.

Se reclutaron 186 mujeres. Se analizan 168 ciclos de 72 mujeres quienes completaron el período de observación de 12 ciclos. Se documentaron un total de 1.358 ciclos.

El control del ciclo menstrual fue adecuado. La duración del mismo, así como la intensidad y la duración de la hemorragia se redujeron ligeramente, permaneciendo dentro de los límites de la normalidad. No se presentaron alteraciones clínicas de importancia como tromboembolismo o trastornos hepáticos. Los cambios en el peso corporal y en los valores de la presión sanguínea no fueron significativos. Las tasas de efectos secundarios y de abandono fueron similares a aquellas informadas para otras píldoras de microdosis.

Se obtuvo un índice de Pearl de 0.12 , lo cual confirma la eficacia y la seguridad anticonceptiva, incluso en los ciclos con olvido de grageas (3.8\%).

Los estudios epidemiológicos a largo plazo permitirán validar la bondad de esta nueva píldora.

PALABRAS ClAVES: Gestodeno, etinilestradiol, píldora anticonceptiva, control del ciclo, aspectos clínicos.

SUMMARY: An open, prospective multicentric study was carried out in Colombia to evaluate efficacy, contraceptive reliability, cycle control and side-effects of the new monophasic contraceptive pill containing $75 \mu \mathrm{g}$ gestodene and $30 \mu \mathrm{g}$ ethinyl estradiol during 12 consecutive cycles.

186 women were recruited. 864 cycles of 72 women who completed the observation period of 12 cycles are analysed. A total of 1358 cycles were recorded.

Cycle control was good, and duration as well as intensity of menstrual bleeding were sligthly decreased, but still within the range of the pretreatment values. Serious adverse events, thromboembolism and hepatic disorders were not observed. Changes in body weight and blood pressure were not significant. Rates of adverse events like headache, nausea, breast tension and discontinuation were similar to those already reported for microdose pills.

With only one pregnancy, the Pearl Index for this study was $\mathbf{0 . 1 2}$, confirming efficacy and contraceptive security, even in cycles with pill omission (3.8\%).

Long term epidemiological studies should validate the benefits of this new pill.

KEY WORDS: Gestodene, ethinyl estradiol, oral contraceptive, cycle control, clinical aspects.

\section{Introducción}

La investigación en anticoncepción hormonal con esteroides sexuales se ha dirigido desde la década de los años sesenta, hacia el desarrollo de nuevos anticonceptivos con el menor contenido hormonal posible sin menoscabar su seguridad anticonceptiva, manteniendo el control del ciclo menstrual y minimizando sus efectos secundarios, y aumentando la seguridad a largo plazo $(1,2)$.

Después de la progresiva reducción en el contenido estrogénico de la píldora, se han investigado nuevos gestágenos cuyas propiedades intrínsecas, androgénicas residuales y antiestrogénicas permitan el desarrollo de anticonceptivos orales con un impacto mínimo sobre el sistema hemostático, el perfil lipídico y el metabolismo de los carbohidratos (3-6).

A la última generación de gestágenos utilizados en los

\footnotetext{
* Departamento de Ginecología - Obstetricia y Reproducción Humana, Fundación Santa Fe de Bogotá, Colombia.

** Departamento Médico, Química Schering Colombiana S.A., Bogotá, Colombia.

•. Versión en Español, con permiso del Editor, de los autores. Aparecido en el J. Endocrinol 1991, 2: 7-22
}

anticonceptivos orales pertenecen el gestodeno, el desogestrel y el norgestimato, relacionados químicamente todos ellos con el levonorgestrel. Sin embargo, el desogestrel y el norgestimato deben ser transformados a metabolitos para su acción biológica total o parcial: el desogestrel a 3-ketodesogestrel y el norgestimato a levonorgestrel (7). El gestodeno en cambio es activo biológicamente en su forma original, presentando como ventaja frente a los compuestos anteriores, que su mayor actividad biológica permite una reducción de la dosis total de esteroides utilizados en la formulación anticonceptiva (8).

Se han realizado varios estudios clínicos con este tipo de preparado anticonceptivo en diferentes países del mundo, principalmente en Europa y en América Latina.

La documentación comprendía un total de 593.455 ciclos analizados en 102.769 mujeres hasta diciembre de 1990 (9-12).

El propósito de realizar este estudio en Colombia fue el de evaluar los aspectos clínicos de una nueva píldora monofásica combinada de etinilestradiol y gestodeno en la anticoncepción oral. 


\section{Material y métodos}

El estudio se llevó a cabo en 7 centros hospitalarios de 4 ciudades colombianas entre abril de 1988 y abril de 1990 . (Tabla No. 1). Se reclutaron 186 mujeres sanas en edad reproductiva, quienes voluntariamente accedieron a participar en un estudio clínico, abierto y prospectivo durante 12 ciclos.

El objetivo era determinar en mujeres colombianas la eficacia, la seguridad, el control del ciclo menstrual y los efectos secundarios de un preparado anticonceptivo monofásico denominado SHD 356 C (Schering, República Federal de Alemania), que contiene 21 grageas compuesta cada una de $75 \mu \mathrm{g}$ de gestodeno y $30 \mu \mathrm{g}$ de etinilestradiol, el cual sería utilizado durante 12 ciclos continuos. Se instruyó a las mujeres para tomar la primera píldora el día 1 del primer ciclo, continuar durante 21 días consecutivos, y reiniciar el tratamiento después de un intervalo de 7 días libre de medicación.

Las voluntarias participantes en el estudio fueron controladas en forma mensual incluyendo la revisión de su calendario menstrual y un cuestionario sobre signos y síntomas experimentados por cada una. Se practicó una citología cervico-vaginal antes de iniciar el estudio, y al 6o. y 12o. ciclo del mismo.

Los criterios de inclusión comprendían mujeres sanas mayores de 18 años en edad reproductiva, con deseo de anticoncepción oral y sin las contraindicaciones usuales para la misma. Fueron excluidas además, aquellas mujeres fumadoras de más de 15 cigarrillos por día y con una edad mayor de 30 años.

Se realiza una descripción del total de ciclos y de los motivos de abandono. Se analizan los 864 ciclos de las 72 voluntarias que lo terminaron, utilizando medidas de tendencia central, de dispersión (media $=\mathrm{X}$ y el error estándar de la media $=\mathrm{SEM}$ ), y de significancia estadística ("t" de Student y chi cuadrado). Se consideró significativa $\mathrm{p}<0.05$.

Tabla No. 1

\section{CIUDADES, CENTROS Y MEDICOS INVESTIGADORES}

\begin{tabular}{|l|l|}
\hline $\begin{array}{l}\text { Barranquilla } \\
\text { - Hospital Metropolitano }\end{array}$ & - G. Acosta - E. Acosta \\
Bogotá & - J. Romero - D. Montenegro \\
- Fundación Santa Fé de Bogotá & - J. Urdinola - J. Galindo \\
- Hospital San José & - L.J. Ruiz - R. Jaramillo \\
- Instituto Materno-Infantil & - A. Lomanto \\
Bucaramanga & - Hospital Universitario R.G.V. \\
$\begin{array}{l}\text { Medellín } \\
\text { - Hospital General }\end{array}$ & G. Jaimes \\
\hline
\end{tabular}

\section{Resultados}

169 mujeres completaron el primer ciclo y $72(42.6 \%)$ de ellas finalizaron los 12 ciclos propuestos (864 ciclos) (Figura No. 1). El número de ciclos incluyendo todas las participantes en el estudio ascendió a 1358.

En relación con la edad, la mayor participación se concentra en las mujeres entre 20 a 39 años (90.3\%), como se aprecia en la Figura No. 2.

Una tercera parte de las mujeres eran nulíparas y dos tercios tenían entre 1 a 3 hijos (Figura No. 3), y la mayoría $(69.4 \%)$ no tenían antecedente de aborto (Figura No. 4).
Figura No. 1

MUJERES VOLUNTARIAS

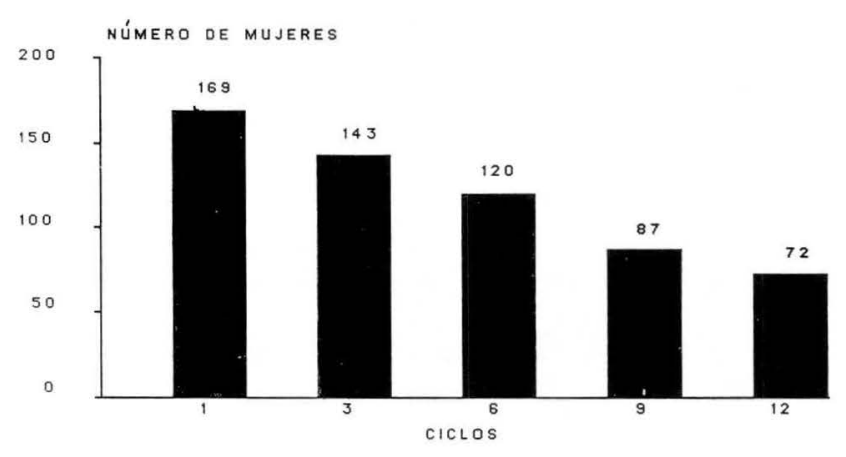

Figura No. 2

DISTRIBUCION POR EDAD $(n=72)$

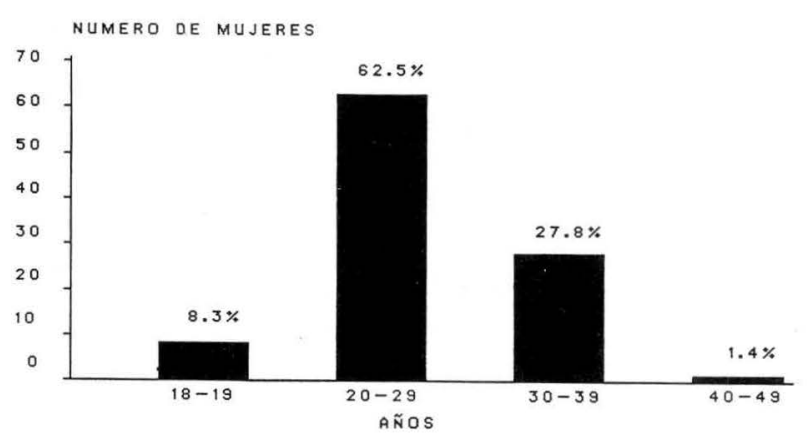

Figura No. 3

PARIDAD $(\mathbf{n}=\mathbf{7 2})$

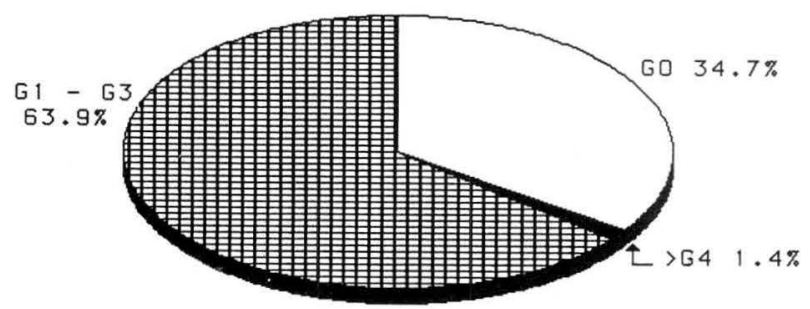

Figura No. 4

ABORTOS PREVIOS

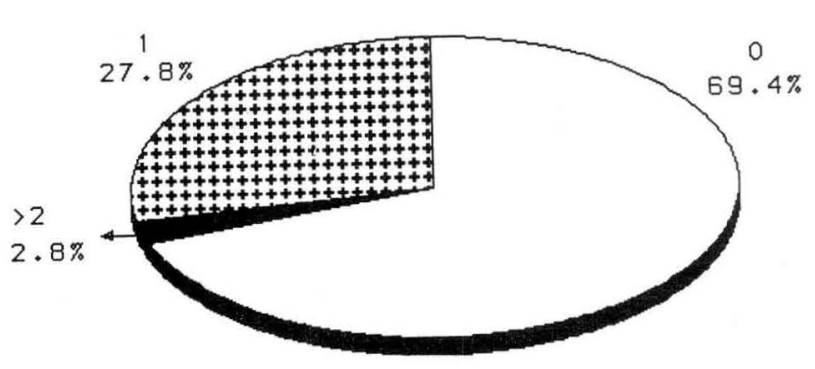

Cerca de la mitad (47.2\%) no había usado anticonceptivos en los 3 meses previos a la iniciación del estudio. Una tercera parte de las mismas eran usuarias anteriores de anticonceptivos orales (Figura No. 5). 
Figura No. 5

ANTICONCEPCION PREVIA $(\mathbf{n}=\mathbf{7 2})$

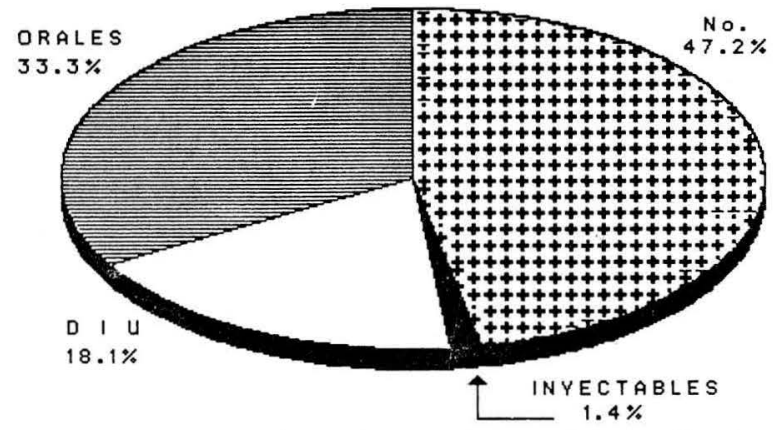

Se presentó un solo embarazo dentro del estudio durante el primer ciclo de tratamiento, sin que se hubiera registrado olvido de alguna de las grageas ni ningún otro factor predisponente. El diagnóstico se realizó durante el ciclo siguiente, y el embarazo terminó en aborto espontáneo.

Sin embargo, no se presentó ninguna gestación a pesar del olvido de una gragea (en 21 ocasiones) o de más de una (una mujer olvidó cuatro grageas cada vez en dos ciclos, cuatro mujeres olvidaron tres grageas en un ciclo y seis mujeres olvidaron dos grageas durante el mismo ciclo). De acuerdo con esto, se produjeron errores en la toma de las grageas en $33(3.8 \%)$ de los 864 ciclos. El índice de Pearl obtenido en relación con 865 ciclos fue de 0.12 .

La duración del ciclo previa al tratamiento, osciló entre 23 a 30 días en el $98.6 \%$ de las mujeres. Tan sólo una (1.4\%) presentaba un ciclo de 34 días, el cual estuvo entre 25 y 28 días durante el tratamiento. La media y el SEM antes del tratamiento fue de $28.8 \pm 1.6$ días. Disminuyó a $26.3 \pm 2$ días durante el primer ciclo, relacionado probablemente con la iniciación de la toma durante el primer día de la menstruación. La duración de los ciclos 3-6-9 y 12 fue de $27.3 \pm 1.7-27.4 \pm 2.2-27.7 \pm 1.5$ y $27.7 \pm 1.2$ días respectivamente. Se presentó diferencia significativa entre el ciclo previo y los ciclos $1-3-6$ y 12 ( $p<0.05)$ (Figuras Nos. 6 y 7).

Figura No. 6

\section{DURACION DEL CICLO}

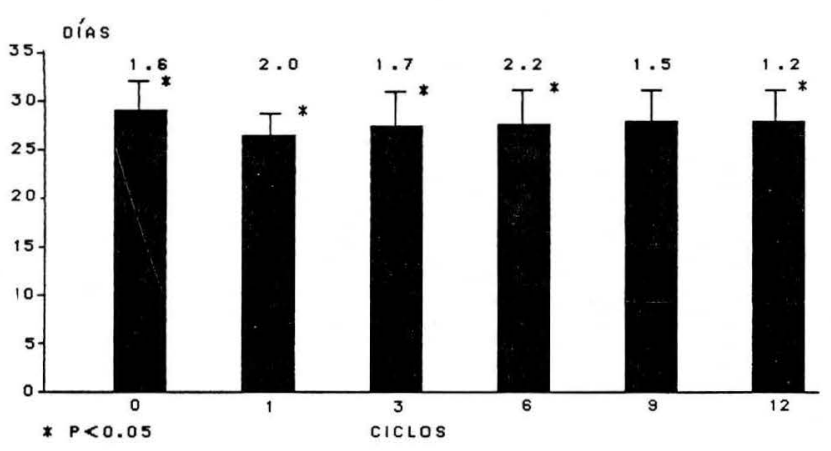

La duración de la hemorragia antes de iniciar el estudio fue de $4.3 \pm 1.5$ días. Se redujo progresivamente y de manera significativa durante los ciclos 1-3-6-9 y 12 a $4 \pm$ $1.4-3.9 \pm 1.1-3.7 \pm 0.9-3.7 \pm 1$ y $3.6 \pm 0.8$ días (p<0.05) (Figuras Nos. 8 y 9).
Figura No. 7

DURACION DEL CICLO (\% DE MUJERES)

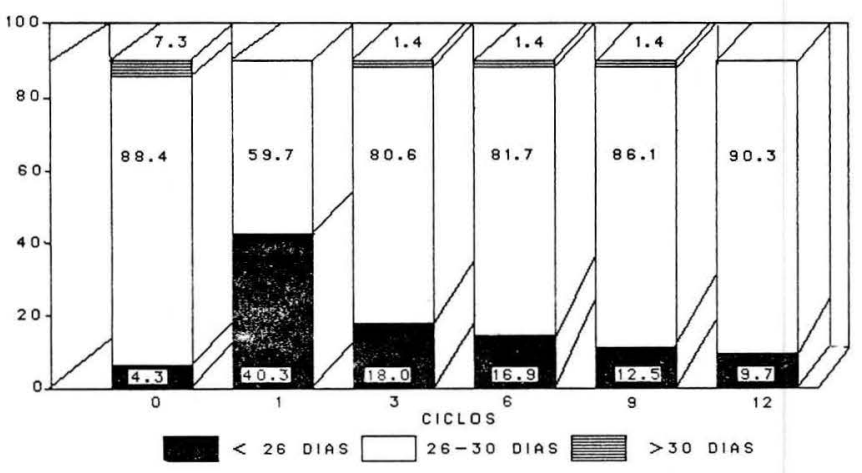

Figura No. 8

DURACION DE LA HEMORRAGIA

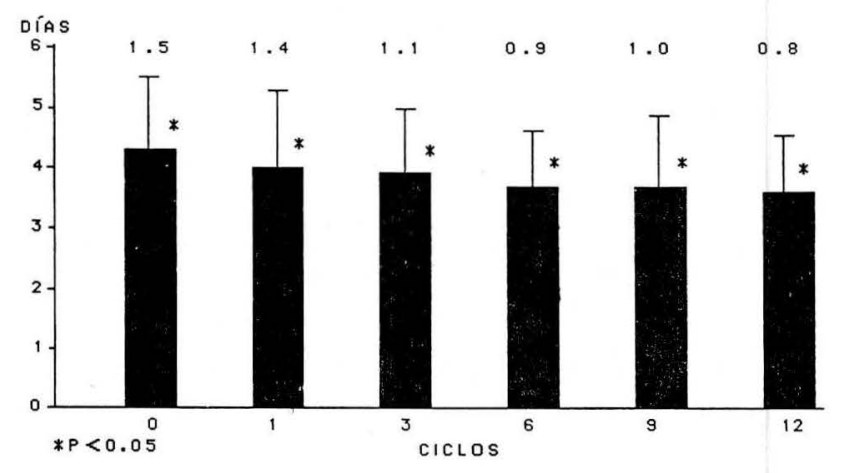

Figura No. 9

DURACION DE LA HEMORRAGIA (\% DE MUJERES)

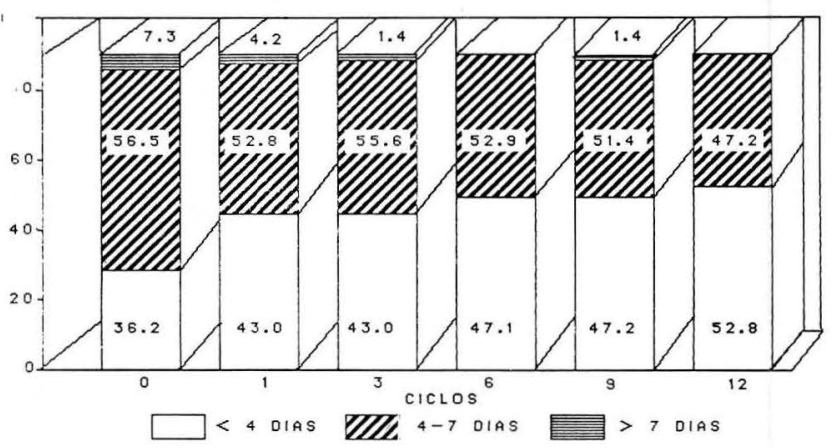

Las mujeres clasificaror. la intensidad de la hemorragia antes de comenzar el estudio como normal en $72.9 \%$.

La clasificación como débil pasó de $11.4 \%$ valor pretratamiento a $9.7 \%-12.5 \%-9.8 \%-11.1 \%$ y $11.1 \%$ en los ciclos 1-3-6-9 y 12. La intensidad fuerte osciló entre $15.7 \%$ pretratamiento y $6.9 \%-4.2 \%-4.2 \%-1.4 \%$ y $4.2 \%$ en los ciclos 1-3-6-9 y 12 respectivamente, siendo significativa esta disminución a partir del tercer ciclo $(\mathrm{p}<0.05)$ (Figura No. 10).

Tres mujeres $(4.2 \%)$ ingresaron en amenorrea postparto. Se presentaron 4 casos diferentes en el primer ciclo $(5.5 \%)$ y uno más ( $1.4 \%$ ) en el ciclo 7 , pero no se presentó ningún caso adicional hasta la finalización del período de observación para un total de $0.6 \%$ de los 864 ciclos analizados. 
Figura No. 10

INTENSIDAD DE LA HEMORRAGIA (\% DE MUJERES)

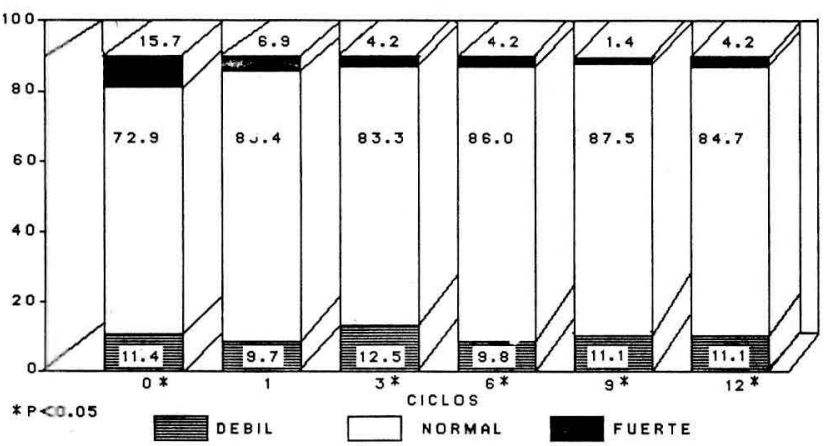

La tasa de hemorragia en forma de manchado fue de $4.8 \%$ y la de hemorragia por disrupción de $1.2 \%$. La presencia simultánea de ambas de $1.2 \%$, para una tasa global de $7.2 \%$ (en 864 ciclos).

En las mujeres que no olvidaron la toma diaria de las grageas, el manchado fue de $5.4 \%$ y la hemorragia por disrupción del $2.4 \%$ (en 831 ciclos). El manchado ascendió a $27.3 \%$ y la hemorragia por disrupción a $9.1 \%$ en las mujeres que olvidaron tomar la píldora (en 33 ciclos) Figura No. 11).

Figura No. 11

\section{MANCHADO Y HEMORRAGIA POR DISRUPCION}

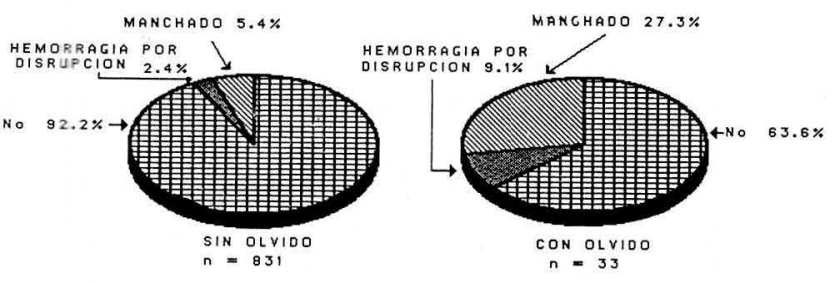

En la tabla No. 2 aparecen las mujeres voluntarias con/sin uso previo de anticonceptivos orales, relacionado con la aparición de manchado, hemorragia por disrupción y la presencia simultánea de ambos. Aunque los porcentajes de manchado y la combinación de este con hemorragia por disrupción son mayores en el grupo sin anticoncepción oral previa, las diferencias no fueron estadísticamente significativas.

Tabla No. 2

HEMORRAGIA INTERMEDIA CON/SIN USO PREVIO DE ANTICONCEPTIVOS ORALES (\% /TOTAL DE CICLOS)

\begin{tabular}{|c|c|c|c|}
\hline & Uso previo & $\begin{array}{l}\text { Sin uso } \\
\text { previo }\end{array}$ & $\begin{array}{l}\text { Tasa } \\
\text { global }\end{array}$ \\
\hline $\begin{array}{l}\text { Manchado } \\
\text { Hemorragia por }\end{array}$ & 1.7 & 6.3 & 4.8 \\
\hline Disrupción & 1.4 & 1.0 & 1.2 \\
\hline Ambos & 0.3 & 1.6 & 1.2 \\
\hline Total & 3.4 & 8.9 & 7.2 \\
\hline Ciclos & 288 & 576 & 864 \\
\hline
\end{tabular}

Como en el caso de otros inhibidores de la ovulación se apreció una disminución significativa de la dismenorrea clasificada como grave a partir del tercer ciclo, pasando de $14.5 \%$ en el ciclo pretratamiento a $4.2 \%$ en el primer ciclo y a $1.4 \%$ en los ciclos 3-6 y 9, desapareciendo al cabo del año $(\mathrm{p}<0.05)$ (Figura No. 12).

Figura No. 12

DISMENORREA (\% DE MUJERES)

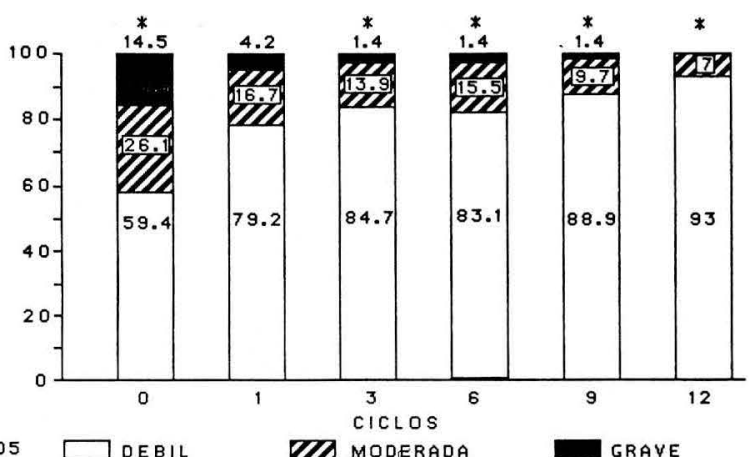

En ninguna de las mujeres voluntarias antes del estudio se evidenciaron valores de tensión arterial por encima de lo considerado como normal $(140 / 90 \mathrm{mmHg})$. Durante el mismo no se presentaron elevaciones mayores a lo referido. En la Figura No. 13 sobre tensión sistólica se aprecia que la $\mathrm{X} \pm$ SEM en los ciclos 0 y 12 fue de $109.5 \pm 2.9$ y de $107 \pm 2.9 \mathrm{~mm} / \mathrm{Hg}$, respectivamente. Se presentó una disminución progresiva que podría interpretarse como significativa $(\mathrm{p}=0.057)$. La tensión diastólica no presentó mayores cambios durante el mismo lapso (Figura No. 14).

Figura No. 13

TENSION SISTOLICA

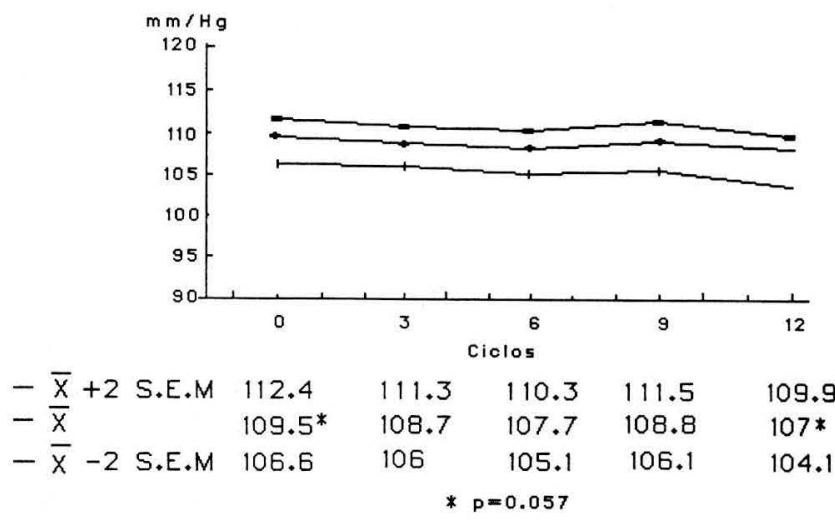

Se utilizó el índice de masa corporal $(\mathrm{p}=\mathrm{kg} /$ talla $=$ $\mathrm{cm}^{2}$ ) para analizar las variaciones del peso corporal. Utilizando la $X \pm 2 S E M$, se aprecia que no hubo variaciones significativas (Figura No. 15). Si se comparan los grupos de bajo peso (índice < 19.1), sobrepeso (25.1-29.9) y obesidad ( $>30)$, se encuentran los siguientes resultados comparando los ciclos 0 y 12: $46.2 \%$ del grupo de bajo peso se 
normalizó, $93.9 \%$ del grupo con peso normal permaneció dentro de esta categoría, $4.1 \%$ de este último grupo pasó al de sobrepeso y $2 \%$ al de bajo peso. $28.6 \%$ de aquellas en el grupo de sobrepeso pasaron a la normalidad. La única mujer obesa permaneció en la misma categoría durante el estudio (Figura No. 16).

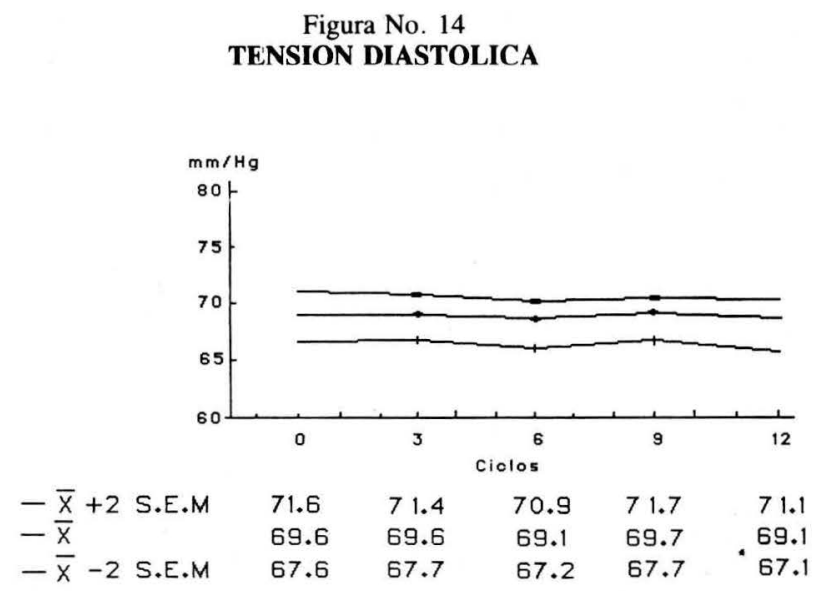

Figura No. 15

INDICE DE MASA CORPORAL

Masa Corporal

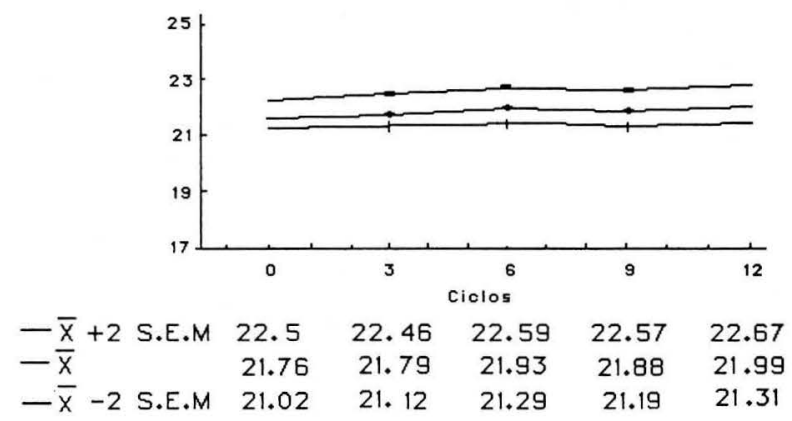

Figura No. 16

INDICE DE MASA CORPORAL ANTES DE TRATAMIENTO Y EN EL CICLO 12

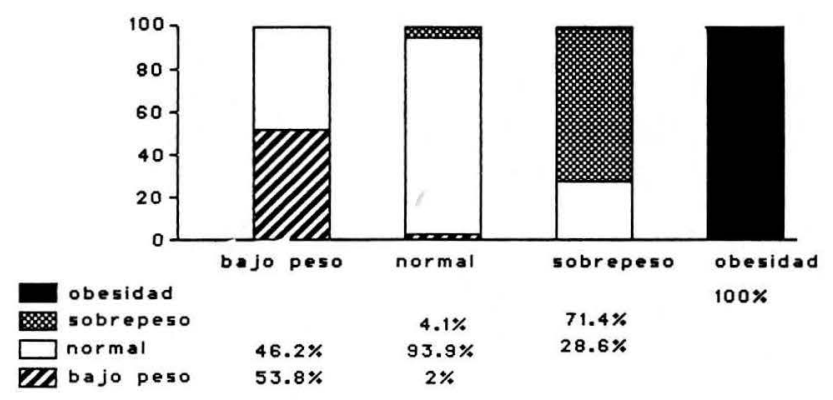

En relación con los efectos secundarios subjetivos (Tabla No. 3), se presentó un aumento significativo $(\mathrm{p}<0.05)$ en la frecuencia de náusea y/o vómito en los ciclos 1 y 9 de tratamiento con relación al ciclo 0 .
Tabla No. 3

EFECTOS SECUNDARIOS SUBJETIVOS (\% DE MUJERES)

\begin{tabular}{|c|c|c|c|c|c|c|}
\hline & \multicolumn{6}{|c|}{ Ciclos } \\
\hline & 0 & 1 & 3 & 6 & 9 & 12 \\
\hline $\begin{array}{l}\text { - Náusea/Vómito } \\
\text { - Mareo } \\
\text { - Tensión Mamaria } \\
\text { - Cefalea } \\
\text { - Migraña } \\
\text { - Nerviosismo } \\
\text { - Depresión } \\
\text { - L Líbido } \\
\text { - Molestias varicosas } \\
\text { - Otros }\end{array}$ & $\begin{array}{r}2.8 \\
1.4 \\
8.3 \\
15.3 \\
1.4 \\
6.9 \\
6.9 \\
- \\
1.4 \\
-\end{array}$ & $\begin{array}{c}11.1^{*} \\
9.7^{*} \\
9.7 \\
6.9 \\
2.8 \\
2.8 \\
1.4 \\
- \\
- \\
5.6\end{array}$ & $\begin{array}{l}8.3 \\
5.6 \\
5.6 \\
9.7 \\
2.8 \\
4.2 \\
2.8 \\
4.2 \\
1.4 \\
4.2\end{array}$ & $\begin{array}{r}4.2 \\
5.6 \\
6.9 \\
11.1 \\
1.4 \\
4.2 \\
2.8 \\
2.8 \\
- \\
6.9\end{array}$ & $\begin{array}{r}12.5^{*} \\
4.2 \\
5.6 \\
11.1 \\
1.4 \\
4.2 \\
2.8 \\
1.4 \\
4.2 \\
1.4\end{array}$ & $\begin{array}{l}8.3 \\
5.6 \\
2.8 \\
9.7 \\
- \\
4.2 \\
5.6 \\
1.4 \\
2.8 \\
-\end{array}$ \\
\hline
\end{tabular}

Se observó igualmente una elevación significativa $(p<0.05)$ en la frecuencia de mareo durante el ciclo 1 de tratamiento. La cefalea se presentó en una alta proporción de mujeres antes de iniciar el tratamiento, sin sufrir variaciones significativas durante el mismo.

Otros síntomas descritos en la Tabla No. 3 no sufrieron variación de importancia. 3 mujeres (casos 26, 67 y 92) presentaron cefalea tipo migraña durante la semana libre de toma de la píldora. En el caso numerado como 26, desde el primer ciclo hasta la conclusión del estudio, en el caso 67 después del segundo ciclo y durante 9 ciclos, y en el caso 92 durante los ciclos 3,10,11 y 12 .

Dentro de los efectos secundarios objetivos observados (Tabla No. 4), el acné presentó una disminución significativa $(p<0.05)$ a partir del ciclo 6 . Las modificaciones en la frecuencia del cloasma no fueron significativas durante este período. No hubo ningún caso de tromboflebitis o de algún trastorno hepático clínico.

Tabla No. 4

EFECTOS SECUNDARIOS OBJETIVOS (\% DE MUJERES)

\begin{tabular}{|l|c|c|c|c|c|c|}
\hline \multirow{2}{*}{} & \multicolumn{7}{|c|}{ Ciclos } \\
\cline { 2 - 7 } & 0 & 1 & 3 & 6 & 9 & 12 \\
\hline . Acné & 11.1 & 11.1 & 4.2 & $2.8^{*}$ & $2.8^{*}$ & $-{ }^{*}$ \\
Cloasma & 8.3 & 8.3 & 6.9 & 8.3 & 12.5 & 9.7 \\
. Edema & 1.4 & - & - & - & - & - \\
Eventos & - & - & - & - & - & - \\
tromboembólicos & - & - & - & - & - & - \\
Trastornos \\
hepáticos
\end{tabular}

En la Tabla No. 5 se anotan los motivos de abandono de 89 mujeres (52.7\% del total). 8 mujeres $(4.7 \%$ ) no completaron los 12 ciclos por el corte hecho para finalizar el estudio para su análisis. El seguimiento no pudo hacerse en una de cada 5 mujeres $(20.7 \%)$. Esta pérdida fue notoria durante los primeros 6 ciclos del estudio. También casi una de cada cinco (19.5\%) de las participantes no lo continuó por diversos motivos médicos, y casi una de cada ocho (12.4\%) por motivos no médicos. Dentro de estos últimos, el deseo de embarazo fue más evidente en la segunda mitad del estudio. En los motivos personales, 2 voluntarias no continuaron a petición del cónyuge. 
Tabla No. 5

MOTIVOS DE ABANDONO*

\begin{tabular}{|c|c|c|c|c|c|c|c|}
\hline \multirow[b]{2}{*}{ CicLo } & \multirow[b]{2}{*}{$\begin{array}{l}\text { EFECTOS } \\
\text { SECUNDARIOS }\end{array}$} & \multirow[b]{2}{*}{$\begin{array}{l}\text { PÉRDIDA DE } \\
\text { SEGUIMIENTOO }\end{array}$} & \multicolumn{4}{|c|}{ Motivos no médicos } & \multirow[b]{2}{*}{ TOTAL } \\
\hline & & & \begin{tabular}{|c|} 
DESEO \\
EMBARAZO
\end{tabular} & \begin{tabular}{|l|} 
CAMBIO \\
MËTODOO
\end{tabular} & \begin{tabular}{|} 
MOTIVOS \\
PERSONALES
\end{tabular} & OTROS & \\
\hline $1-5$ & 13 & 15 & & 1 & 3 & & $\begin{array}{c}32 \\
(18.9 \%)\end{array}$ \\
\hline $4-8$ & 16 & 10 & 1 & & 2 & 2 & $\begin{array}{c}31 \\
(18.3 \%)\end{array}$ \\
\hline $7-12$ & 4 & 10 & 5 & 2 & 3 & 2 & $\begin{array}{l}28 \\
(15.4 \%)\end{array}$ \\
\hline $\begin{array}{l}\text { TOTaL } \\
1-12\end{array}$ & $\begin{array}{c}33 \\
(19.5 \%)\end{array}$ & $\begin{array}{c}35 \\
(20.7 \%)\end{array}$ & $\begin{array}{c}6 \\
(3.6 \%)\end{array}$ & $\begin{array}{c}3 \\
(1.8 \%)\end{array}$ & $\begin{array}{c}8 \\
(12.4 \%) \\
(4.7 \%)\end{array}$ & $\begin{array}{c}4 \\
(2.4 \%)\end{array}$ & $\begin{array}{c}89 \\
(52.7 \%)\end{array}$ \\
\hline
\end{tabular}

Los efectos secundarios como causa de abandono fueron más frecuentes durante los 6 primeros ciclos. En la Tabla No. 6 aparece que la cefalea, la náusea y/o el vómito fueron los síntomas más frecuentemente informados.

Las causas objetivas de abandono (Tabla No. 7) tuvieron una baja frecuencia. La hemorragia intermenstrual fue causa de abandono en sólo 3 pacientes (1.8\%), 2 casos de hemorragia por disrupción en el ciclo 1 y un caso de manchado durante el ciclo 4.

Tabla No. 6

CAUSAS SUBJETIVAS DE ABANDONO *CASOS REPORTADOS

\begin{tabular}{|c|c|c|c|c|c|}
\hline $\begin{array}{l}\text { Causas Ciclos } \\
\text { de abandono }\end{array}$ & $1-2$ & $3-4$ & $5-6$ & $7-8$ & $9-12$ \\
\hline $\begin{array}{l}\text { Causas subjetivas } \\
\text { 1. Cefalea } \\
\text { 2. Náusea/vómito } \\
\text { 3. Irritabilidad - } \\
\text { nerviosismo } \\
\text { Depresión }\end{array}$ & $\begin{array}{l}3 \\
3\end{array}$ & $\begin{array}{l}7 \\
5 \\
4\end{array}$ & $\begin{array}{l}4 \\
3 \\
2\end{array}$ & 2 & \\
\hline $\begin{array}{l}\text { 4. Molestias } \\
\text { varicosas } \\
\text { 5. Tensión mamaria } \\
\text { 6. Otras }\end{array}$ & $\begin{array}{l}1 \\
1 \\
1\end{array}$ & 1 & $\begin{array}{l}2 \\
1 \\
3\end{array}$ & 1 & 1 \\
\hline
\end{tabular}

Tabla No. 7

CAUSAS OBJETIVAS DE ABANDONO

\begin{tabular}{|l|c|c|}
\hline & $\begin{array}{c}\text { Casos } \\
\text { reportados }\end{array}$ & $\%$ ' 169 \\
\hline - Hemorragia Intermenstrual & 2 & 1.8 \\
Hemorragia por disrupción & 1 & \\
Manchado & 3 & 1.8 \\
- Aumento de peso & 1 & 0.6 \\
- Embarazo & 1 & 0.6 \\
\hline - Otros & 1 & \\
\hline
\end{tabular}

El aumento de peso fue la razón argumentada por 3 mujeres en los ciclos 3 y 4 , pero el índice de masa corporal no mostró variación por fuera de los valores normales.

Otros motivos de abandono que se informaron fueron tensión mamaria, epigastralgia en 2 casos, disminución de la líbido en 2 casos, sequedad vaginal en uno, dismenorrea en uno y un caso de candidiasis vaginal.
Una mujer fue excluida del estudio durante el ciclo 2, al comprobarse que había ingresado embarazada, confundiéndose al ingreso la hemorragia por amenaza de aborto como la menstruación. Su embarazo y parto cursaron normalmente con un recién nacido sano.

\section{Discusión}

Por primera vez se realiza en Colombia un estudio clínico, abierto y multicéntrico con la nueva píldora anticonceptiva que contiene etinilestradiol y un nuevo gestágeno, el gestodeno. Es importante tener en cuenta esto, en un país donde la píldora figura como el primer método reversible, comprendiendo 714.000 usuarias y un consumo estimado de 10 millones de ciclos por año (13).

A pesar de la presentación de un embarazo dentro del estudio, no relacionado con error en la toma del preparado, el índice de Pearl obtenido es comparable con el rango usual, de 0.03 a 0.22 , para los anticonceptivos orales combinados de microdosis (14). Se destaca que la seguridad anticonceptiva no se vio afectada por errores en la toma de la píldora en $3.8 \%$ de los ciclos analizados.

La duración del primer ciclo disminuyó debido a la recomendación actual de iniciar la toma del primer ciclo del preparado el primer día de la menstruación (15-17).

El acortamiento significativo de la duración del ciclo durante el período de observación, conservándose aún dentro del rango aceptado como normal, está probablemente relacionado con la eficaz inhibición de la secreción de gonadotropinas y secundariamente de estradiol (18). Se ha informado en un estudio realizado por Thomas y Vankrieken, que tan sólo unas pocas voluntarias presentaron maduración folicular durante el intervalo de 7 días libres de la toma de la píldora (19). Podría asumirse entonces, que la duración del ciclo y la posterior hemorragia menstrual dependen del grado de supresión hormonal.

La reducción progresiva y significativa que se observó de la duración y la intensidad de la hemorragia menstrual, es un efecto bien conocido de los anticonceptivos orales especialmente con los de microdosis, debido a la menor estimulación hormonal sobre el endometrio $(20,21)$. Este hecho se acepta en la actualidad como uno de los beneficios no anticonceptivos de la píldora (1).

La tasa de amenorrea fue muy baja, $0.6 \%$ de los 864 ciclos analizados, comparable con la informada en estudios hechos en América Latina y Europa $(22,23)$.

La dismenorrea clasificada como grave desapareció al cabo de un año de tratamiento, aunque el preparado utilizado posee el contenido hormonal más bajo disponible en la actualidad, siendo este efecto similar al de otros compuestos de microdosis (24).

Aunque existen informes sobre elevación de la presión arterial inducida por los anticonceptivos orales $(23,25)$, también se ha observado la normalización de valores diastólicos ligeramente elevados $(90-105 \mathrm{mmHg}$ ) al utilizar la píldora con gestodeno. Además, experimentalmente se ha documentado una acción antimineralocorticoide del gestodeno $(23,26)$. En este estudio no se incluyeron sin embargo mujeres con hipertensión arterial; tampoco se presentaron durante el mismo valores tensionales por encima de lo normal. Se presentó una disminución de la tensión arterial sis- 
tólica que podría interpretarse estadísticamente como significativa, $\mathrm{p}=0.057$, sin que por esta razón pueda postularse un significado clínico cierto o que exista una relación de causalidad con el gestodeno.

Los resultados del comportamiento del índice de masa corporal relacionado con el peso fueron similares a los obtenidos por otros autores en México (15), sugiriendo que el efecto anabólico de esta nueva píldora, apreciado desde este punto de vista, no es clínicamente relevante. Debe recordarse que existen muchos otros factores que también intervienen en la variación del peso corporal.

Con respecto a los efectos secundarios subjetivos, estos son similares a los observados en un estudio europeo multicéntrico (28).

Esta nueva píldora reduce también la tasa de acné durante el período de tratamiento. A pesar de la localización geográfica tropical de Colombia, el cloasma no se modificó durante el estudio.

No se presentó ninguna complicación tromboembólica, hecho ya corroborado en otros 2 estudios en América Latina $(15,22)$.

La no continuación de la píldora anticonceptiva llega a ser hasta del $50 \%$ al cabo de un año de utilización, incluso en países como Estados Unidos y Gran Bretaña $(26,29)$.
Esta suspensión obedece en la mayoría de los casos a razones no médicas (30), siendo estas observaciones también válidas para el presente estudio.

\section{Conclusiones}

Los resultados obtenidos confirman la eficacia y la seguridad anticonceptiva de la nueva píldora anticonceptiva combinada con gestodeno. incluso en los ciclos con olvido de grageas.

El control del ciclo menstrual es adecuado. La duración del mismo así como la intensidad y la duración de la hemorragia se ven ligeramente disminuidas, pero permaneciendo dentro de los límites de la normalidad.

No se presentaron alteraciones clínicas de importancia y la frecuencia de efectos secundarios fue baja.

Los estudios epidemiológicos a largo plazo permitirán validar la bondad de esta nueva píldora.

\section{Agradecimientos}

Al Dr. Edgard Rodríguez por la asesoria estadística, y a Amanda Vargas, Nohemy Coy y Martha Lucía Bernal por su labor de secretaria.

Este trabajo contó con el apoyo de Química Schering Colombiana S.A.

\section{BIBLIOGRAFIA}

1. World Health Organization: Steroid contraception and risk of neoplasia. WHO Tech Rep Ser 1978; 619.

2. Consensus Development Meeting: Metabolic aspects of oral contraceptives of relevance for cardiovascular diseases. Am. J. Obstet. Gynecol. 1990; 162: 1335-1337.

3. Hoppe G. Gestoden, an innovative progestogen. Adv Contracept 1987; 3: 159-166.

4. Jespersen J., Petersen KR., Skouby SO. Effects of newer oral contraceptives on the inhibition of coagulation and fibrinolysis in relation to dosage and type of steroid. Am. J. Obstet. Gynecol. 1990; 163: 396-403.

5. Gevers JA., Dersjant-Roorda MC., Helmerhorst FM., de Boer R., Neymeyer-Leloux A., Havekes L. Estrogenic effect of gestodeneor desogestrel-containing oral contraceptives on lipoprotein metabolism. Am. J. Obstet. Gynecol. 1990; 163: 358-362.

6. Gaspard UJ., Lefebvre PJ. Clinical aspects of the relationship between oral contraceptives, abnormalities in carbohydrate metabolism and the development of cardiovascular disease. Am J. Obstet. Gynecol. 1990; 163: 334-343.

7. Chez RA. Clinical aspects of three new progestogens: Desogestrel, gestodene and norgestimate. Am. J. Obstet. Gynecol. 1989; 160: 1296-1300.

8. Spona J., Huber J. Pharmacological and endocrine profiles of gestodene. Int. J. Fertil. 1987; Suppl 32: 6-14.

9. Düsterberg B., Brill K., Muehe B. El contraceptivo oral monofásico con gestodeno: revisión y resultados del programa mundial de ensayos clínicos. En: Elstein M. ed. Un nuevo progestágeno específico para la contracepción oral a dosis bajas. Carnforth: The Parthenon Publishing Group Ltd., 1989; 45-55.

10. Aldrighi JM., Carter R. Primeras experiencias clínicas en América Latina con el nuevo contraceptivo oral conteniendo gestodeno. En: Elstein M., ed. Un nuevo progestágeno específico para la contracepción oral a dosis bajas. Carnforth: The Parthenon Publishing Group Ltd., 1989; 89-97.

11. Rabe T., Runnebaum B., Kohlmeier M., Harenberg J., Weicker H., Unger R. Clinical and metabolic effects of gestodene and levonorgestrel. Int. J. Fertil. 1987; Suppl 32: 29-44.
12. Düsterberg B., Brill K. Clinical experience with a low-dose oral contraceptive containing gestodene. Adv. Contracept. 1990; Suppl 6: $37-50$

13. Profamilia y DHS: Encuesta de Prevalencia, Demografía y Salud 1990. Informe preliminar. Profamilia, Bogotá, Octubre 1990.

14. Taubert HD., Kuhl H. En: Kontrazeption mit Hormonen. Stuttgart \& New York: Georg Thieme Verlag. 1981; 194.

15. Martínez M., Pérez-Palacios G., Garza-Flores J., Bassol S., Recio R. Efectividad y seguridad de un nuevo preparado anticonceptivo oral combinado 75 mcg gestodeno más 30 mcg etinilestradiol en mujeres mexicanas. Investigación Médica Internacional 1990; 17: 3-9.

16. Percival-Smith RKL., Yuzpe AA., Desrosiers JA., Rioux JE., Guilbert E. Cycle control on low-dose oral contraceptives: A comparative trial. Contraception 1990; 42: 253-262.

17. Stickland J., Tolowinska IY., Anthony F., Dennis KJ. The suppression of ovarian function by the new oral contraceptive-Femodene. Contraception 1987; 35(5): 447-456.

18. Spona J., Huber J. Inhibition of ovulation with gestodene. En: Elstein M., ed. Gestodene, development of a new gestodene-containing lowdose oral contraceptive. Carnforth: The Parthenon Publishing Group Ltd., 1987; 45-54.

19. Thomas K., Vankrieken I. Inhibición de la ovulación mediante un anticonceptivo oral monofásico con gestodeno. En: Aldrighi J, Urdinola $\mathbf{J}$, eds. Hacia un nuevo estándar en la anticoncepción oral. Barcelona: Editorial Doyma, 1990; 45-54.

20. Chantler EN., Sharma R., Eyong E., Elstein M. Endometrial receptor response to two progestogens, levonorgestrel and gestodene. En: Elstein M., ed. Gestodene, development of a new gestodene-containing low-dose oral contraceptive. Carnforth: The Parthenon Publishing Group Ltd., 1987; 67-71.

21. Haboubi NY., Eyong E., Chantler EN., Elstein M. Comparative histology of the endometrium under treatment with levonorgestrel and gestodene. En: Elstein M., ed. Gestodene, development of a new gestodene-containing low-dose oral contraceptive. Carnforth: The Parthenon Publishing Group Ltd., 1987; 73-79.

22. Aldrighi JM., Carter R. Primeras experiencias clínicas en América Latina con el nuevo contraceptivo oral conteniendo gestodeno. En: 
Elstein M., ed. Un nuevo progestágeno específico para la contracepción oral a dosis bajas. Carnforth: The Parthenon Publishing Group Ltd., 1989; 89-97.

23. Mall-Haefeli M. Biochemical and clinical results of a new low-dose oral contraceptive. En: Breckwoldt M., Duesterberg B., eds. Gestodene, a new direction in oral contraception. Carnforth: The Parthenon Publishing Group Ltd., 1988; 69-86.

24. Milson I., Sundell G., Andersch B. The influence of different combined oral contraceptives on the prevalence and severity of dysmenorrhea. Contraception 1990; 42(5): 497-506.

25. Royal College of General Practitioners: Oral contraceptives and health. New York: Pitman Publishing, 1974.

26. Kirkman RJ. Interim results of a UK study comparing a new monophasic pill containing gestodene to Microgynon $30(\mathrm{R})$ and proceeding to open assessment. En: Elstein M., ed. Gestodene, development o f a new gestodene-containing low-dose oral contraceptive. Carnforth: The Parthenon Publishing Group Ltd., 1987; 91-104.

27. Losert W., Casals-Stenzel J., Buse M. Progestogens with antimineralocorticoid activity. Drug Res 1985; 35(I), 2: 459-471.

28. Unger R. Clinical investigations with a new gestodene-containing oral contraceptive (Femodene). En: Elstein M., ed. Gestodene, development of a new gestodene-containing low-dose oral contraceptive. Carnforth: The Parthenon Publishing Group Ltd., 1987; 81-89.

29. Adams Hillard PJ. The patient's reaction to side effects of oral contraceptives. Am. J. Obstet. Gynecol. 1989; 161: 1412-1415.

30. Combined Oral Contraceptives. En: Breedlove B., Judy B., Martín N., eds. Contraceptive Technology 1988-1989-14th Revised Edition. Atlanta: Irvington Publishers Inc., 1988; 194-250.

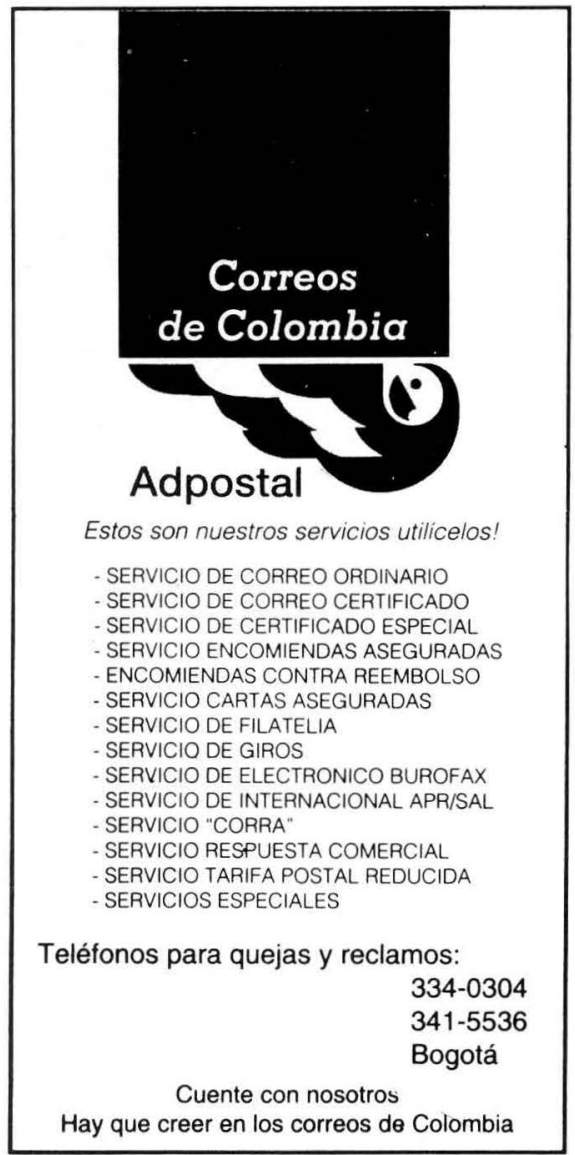

